\title{
THE ABSOLUTE MAGNITUDE OF RR LYRAE STARS
}

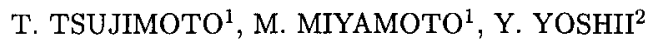 \\ 1 National Astronomical Observatory, Mitaka, Tokyo 181, Japan \\ 2 Institute of Astronomy, University of Tokyo, Mitaka, Tokyo 181, Japan
}

The present determination of the absolute magnitude $M_{V}(\mathrm{RR})$ of RR Lyrae stars is twofold, relying upon Hipparcos proper motions and trigonometric parallaxes separately. First, applying the statistical parallax method to the proper motions, we find $\left\langle M_{V}(\mathrm{RR})\right\rangle=0.69 \pm 0.10$ for 99 halo RR Lyraes with $\langle[\mathrm{Fe} / \mathrm{H}]>=-1.58$. Second, applying the Lutz-Kelker correction to the RR Lyrae HIP95497 with the most accurately measured parallax, we obtain $M_{V}(\mathrm{RR})=0.57-0.74$ at $[\mathrm{Fe} / \mathrm{H}]=-$ 1.6. Furthermore, allowing full use of low accuracy and negative parallaxes as well for $125 \mathrm{RR}$ Lyraes with $-2.49 \leq[\mathrm{Fe} / \mathrm{H}] \leq 0.07$, the maximum likelihood estimation yields the relation, $M_{V}(\mathrm{RR})$ $=(0.59 \pm 0.37)+(0.20 \pm 0.63)([\mathrm{Fe} / \mathrm{H}]+1.60)$, which formally agrees with the recent preferred relation. The same estimation yields again $M_{V}(\mathrm{RR})=0.65 \pm 0.33$ for the 99 halo RR Lyraes. Although the formal errors in the latter two estimates are rather large, all of the four results suggest the fainter absolute magnitude, $M_{V}(\mathrm{RR})=0.6-0.7$ at $[\mathrm{Fe} / \mathrm{H}]=-1.6$. The present results still provide the lower limit on the age of the universe which is inconsistent with a flat, matter-dominated universe and current estimates of the Hubble constant.

\section{SUGGESTION TO REVISE THE HIPPARCOS-PARALLAX OF CEPHEIDS BELONGING TO BINARY SYSTEMS}

\author{
L. SZABADOS \\ Konkoly Observatory, P.O. Box 67, H-1525, Budapest XII, Hungary
}

It has been suspected (Szabados 1997) that the apparent orbit of previously unresolved binaries involving a Cepheid component falsifies the Hipparcos-parallaxes deduced for these Cepheids. Since the incidence of binaries among Cepheids exceeds 50 per cent, this adverse effect on the parallax determination is a major error source.

An obvious remedy is to repeat the determination of the five astrometric parameters for the Cepheids, members in binary systems, taking into account the orbital elements known from spectroscopic studies. This will result in more precise parallaxes and cosmic distance scale. Moreover, these Cepheids are ideal targets for the future microarcsec astrometric missions.

This study was partly supported by OTKA-grants T 014852 and T 022946.

\section{References}

Szabados L., 1997, in Proc. Conf. Hipparcos Venice'97; ESA SP-402, p. 631 